\title{
บUisuersily
}

\section{EMOHEX: An eye tracker based mobility and hand exoskeleton device for assisting disabled people}

Meena, YK., Chowdhury, A., Cecotti, H., Wong-Lin, K., Dutta, A., \& Prasad, G. (2017). EMOHEX: An eye tracker based mobility and hand exoskeleton device for assisting disabled people. In Unknown Host Publication IEEE Xplore. https://doi.org/10.1109/SMC.2016.7844553

Link to publication record in Ulster University Research Portal

\section{Published in:}

Unknown Host Publication

Publication Status:

Published online: 09/02/2017

DOI:

10.1109/SMC.2016.7844553

\section{Document Version}

Author Accepted version

\section{General rights}

Copyright for the publications made accessible via Ulster University's Research Portal is retained by the author(s) and / or other copyright owners and it is a condition of accessing these publications that users recognise and abide by the legal requirements associated with these rights.

\section{Take down policy}

The Research Portal is Ulster University's institutional repository that provides access to Ulster's research outputs. Every effort has been made to ensure that content in the Research Portal does not infringe any person's rights, or applicable UK laws. If you discover content in the Research Portal that you believe breaches copyright or violates any law, please contact pure-support@ulster.ac.uk. 


\title{
EMOHEX: An Eye Tracker based Mobility and Hand Exoskeleton Device for Assisting Disabled People
}

\author{
Y. K. Meena*, A. Chowdhury ${ }^{\dagger}$, H. Cecotti*, K. Wong-Lin*, S. S. Nishad ${ }^{\dagger}$, A. Dutta ${ }^{\dagger}$ and G. Prasad* \\ ${ }^{*}$ Intelligent System Research Centre, Ulster University, Magee Campus, Derry Londonderry, N. Ireland, UK \\ †IIT Kanpur, Kanpur, India
}

\begin{abstract}
People suffering from a variety of upper and lower limb disabilities due to different neuro-muscular diseases or injuries, often find it difficult to perform day-to-day activities of mobility and grasping (pick and place) objects. This paper presents the feasibility and utility of a newly developed assistive device named EMOHEX, for disabled people to perform some activities of daily living (ADL). EMOHEX is an integrated platform that combines a low cost eye-tracking device with a powered-wheelchair mounted hand-exoskeleton, which can assist disabled people in grasping objects while moving around. A dual control panel based graphical user interface is designed wherein the user's intention to select any command button is detected through eye-tracking. The dual control consists of wheelchair control panel and exoskeleton control panel, which are interchangeable by a switch button common to both the panels. The hand-exoskeleton is capable of assisting grasp, hold, and release action. Experiments conducted on $\mathbf{1 6}$ healthy participants revealed that performance metrics were significantly $(\mathbf{p}<0.01)$ similar for the same task complexity while for different task complexities the performance metrics were significantly $(\mathbf{p}<\mathbf{0 . 0 1})$ different across all the participants. These results showed the feasibility and stability of the system, respectively. Moreover, the information transfer rate (ITR) of eye-tracker was found satisfactory at $55.28 \pm 1.29 \mathrm{bits} / \mathrm{min}$ and $51.02 \pm 1.72 \mathrm{bits} / \mathrm{min}$ for simple and complex task, respectively. Thus, EMOHEX has the potential as a quality assistive device for disabled people.
\end{abstract}

\section{INTRODUCTION}

People suffering from different neuromuscular diseases, traumatic injuries in their central or peripheral nervous system face several problems in carrying out most common daily life activities such as locomotion and handling household objects [1]. A large number of assistive technologies have been developed to assist these people among which the powered wheelchairs and hand-exoskeletons are the newer trends [2]. Researchers have exploited different modalities to control such devices like kinetic sensors, electroencephalogram (EEG) and electromyogram (EMG) signals, eye-tracking etc. Some of the earlier control devices for powered wheelchairs includes joysticks, sip-puff control [3]. But joystick based controls are not suited for people with hand disability and sip-puff control is non-intuitive. Therefore multimodal controls combining eyetracking, voice command and touch have been explored [4]. For example, a combination of head movement controller and brain-computer interface (BCI) has also proved to be efficient for navigation using smart wheelchair [5]. New free-view solutions for gaze based wheelchair navigation have also been introduced for faster operation [6]. An eye-tracking device in combination with scanning based techniques can increase users comfort and speed of interaction [7], [8]. BCI devices are also coupled with vision based eye-tracking devices for rehabilitation of users' upper-extremity where the user intentions are decoded using the eye-tracking device and the continuous control of the exoskeleton is achieved with motorimagery based BCI [9]. Virtual reality based serious games are also developed using the combination of eye-tracker and haptic feedback for rehabilitation purposes [10].

Exoskeletons have found a vast range of applications in different domains of assistive and rehabilitative technologies. Leg-exoskeletons (e.g., ALEX) are used in neuro-motor training of gait [11]. Rice-wrist system has also been developed for the functional recovery of wrist and forearm [12]. Exoskeletons are also tested for individual muscle activation [13] and systems like CAFE have been developed for individual joint level restoration of finger-mobility following stroke [14]. Studies have indicated that there is utmost need for lowcost devices for the activities of ADL in home setting [15]. Among different modalities used for controlling such devices, $\mathrm{BCI}$ is promising and has become a nearly matured field for building practical applications [16]. Adaptive hybrid BCI using a combination of EEG and EMG has been successfully implemented to control hand exoskeleton [17]. However it suffers from poor accuracies due to several factors including lack of concentration, stress, and environmental noise in a real-world scenario [18], [19]. On the other hand as human gaze behavior is strongly correlated to user intentions, and has the potential to generate a large number of commands, eye-tracking devices have become a popular choice as an input modality. The absolute point of gaze (POG) can be tracked through several techniques like visual-oculography (VOG), infrared-pupil corneal reflection (PCR) and electro-oculography (EOG) [20]. With the advent of low-cost and high precision eye-tracking devices several applications like replacing the mouse in the GUI environment and controlling robotic devices have been implemented successfully [21]. But there is a need to develop robust assistive devices which solves two major purposes of ADL that are locomotion and object grasp. To overcome these 


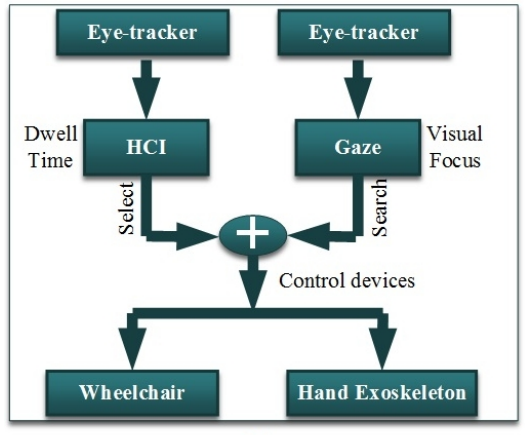

(a)

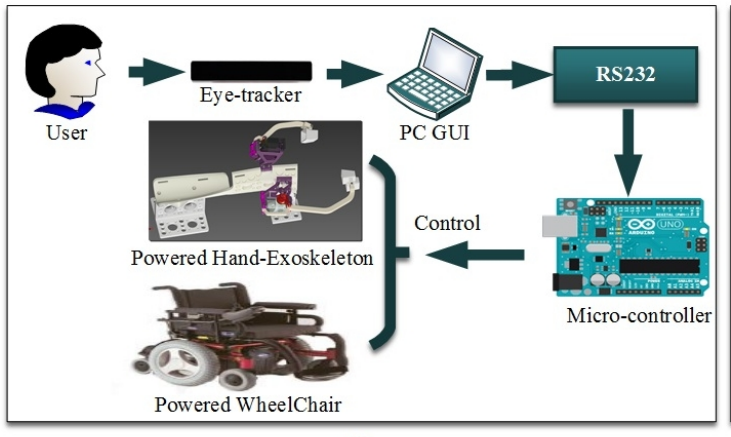

(b)

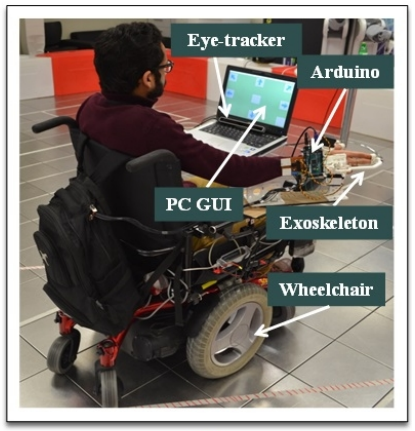

(c)

Fig. 1. System overview. (a) Proposed model of the system where an item search and selection is done by gaze and dwell time respectively, to control the wheelchair and the hand exoskeleton, (b) Block diagram of the proposed system, (c) Organization of the system components.

shortcomings, we have explored the possibility of integrating a low-cost eye-tracking device (Eye-Tribe [22]) with a poweredwheelchair mounted hand-exoskeleton which we have termed as EMOHEX. The objective of the experiment is to study two aspects of the proposed EMOHEX system (see Fig. 1 $(\mathrm{a}-\mathrm{c}))$. The first is to see whether the system is feasible to be used by any participant with healthy eye condition (i.e., person should not be suffered from gaze abnormalities such as gaze palsy, astigmatism etc.). The second is to find out stable performance metrics which are correlated with the complexity of the task. The system performance, evaluated on the basis of performance metrics, shows that the system is highly potent to be used in the real world application. This paper is organized as follows: Section II discusses the experimental paradigm which includes the overview of the system along with the experimental protocol. Section III is dedicated to the methods used. Section IV presents the results. Section V discusses the significance of the work, and Section VI gives the conclusion.

\section{EXPERIMENTAL PARADIGM}

\section{A. System overview}

As shown in Fig. 1 (a-c), the developed system consists of three major components. The first is a human computer interface (HCI) based on eye-tracking. This is composed of a low-cost eye-tracking device called the Eye-Tribe [23] to capture the POG of the user and translate it into screen coordinates of a visual-display-unit (VDU) of a laptop, where the combined control panel for the wheelchair navigation and exoskeleton control is displayed. The co-ordinates are then used to select different commands in the control panel. The second component is a powered wheelchair with two active and four passive wheels. The active wheels are driven in differential mode for turning. The third component is a two finger (thumb and coupled index-middle fingers) handexoskeleton which has one degree of freedom in each finger for assisting the user to do grasp, hold, and release action. The hand-exoskeleton is mounted on the right arm of the wheelchair on a movable arm rest and the laptop is attached in front of the wheelchair on a horizontal platform. The eyetracker is attached at the bottom of the VDU facing the direction of the users eyes. The user can control the wheelchair and the exoskeleton by using the combined control panel and can perform certain tasks of locomotion and grasp. The complete overview of the system is depicted in Fig. 1.

\section{B. Participants}

Sixteen consenting healthy male volunteers participated in the study $($ mean $=28.6$, standard deviation $=2.07$, age range 20-32 years), all right handed. No participants had prior experience of using an eye-tracker or a hand-exoskeleton or a wheelchair. The participants were advised about the purpose and nature of the study. No financial benefit for the participant was associated with the experiments. The Helsinki Declaration of 2000 was followed while conducting the experiments.

\section{Experimental protocol}

The experimental protocol is designed to establish the feasibility of such a system in terms of testing all the functionalities and user interactions associated with the system. The basic functionalities of this system are navigations such as moving forward (F), backward (B), left (L), right (R), forward-left (FL), and forward-right (FR) and object handling such as grasping an object, holding it and dropping or releasing it. The test was designed using two different tracks varying in terms of complexity (see in Fig. 2) where the users need to perform an object grasping and releasing task while following the track from the starting to ending position. There was a source location on the track where the object was initially placed. The user needs to grab the object from that source location and hold it. Then, it is taken to the destination location while following the track and the object is dropped in a bin. After that the user follows the rest of the track to reach the ending location. The simple and complex track layouts and the different views of a participant undergoing the test are shown in Fig. 3 (a-c).

During the tests the user interactions with the HCI were logged for analyzing the performance of the users in different experimental trials. A 9-point calibration scheme was applied to calibrate the eye-tracker prior to each experiment to estimate accurate POG. No pre-training session is required for the users. A population of 16 participants has been taken and divided 


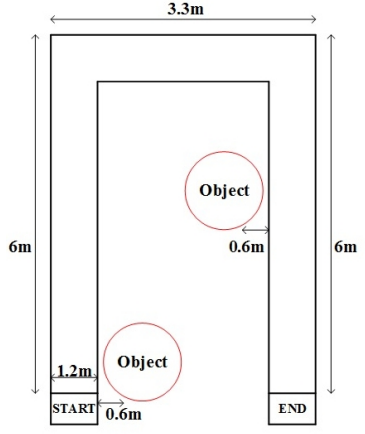

(a)

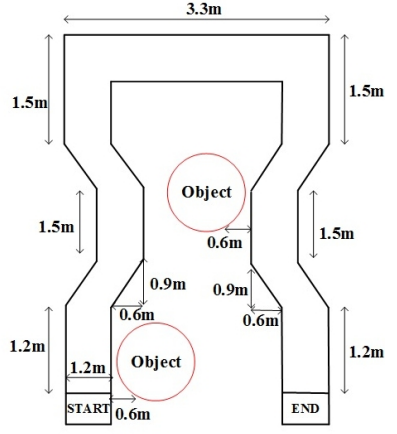

(b)
Fig. 2. Schematic view of the track. (a) simple, (b) complex.

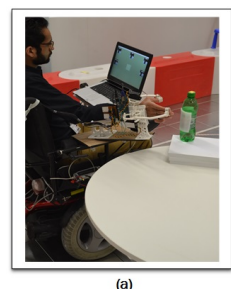

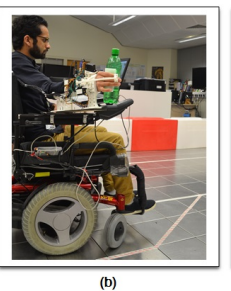

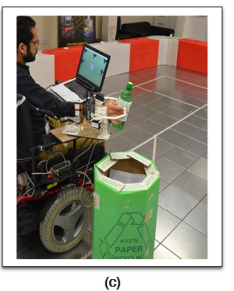

Fig. 3. Participant performing different tasks on the track, (a) grabbing the object at source location, (b) holding and carrying it to destination, and (c) dropping the object to the destination.

into two groups of 8 . We have conducted the experiments on the simple track with one group and on complex track with the other group. The user interaction log after each of these experiments was kept in a database for the comparative study of system and user performances between these two test conditions.

\section{Data acquisition}

The EyeTribe eye-tracker was used to record the eye gaze signals at $30 \mathrm{~Hz}$ sampling rate. It involves binocular infrared illumination with spatial resolution $\left(0.1^{\circ}\right.$ (RMS)), which records $(\mathrm{x}, \mathrm{y})$ screen coordinate and pupil diameter in $\mathrm{mm}$.

\section{METhodS}

\section{A. Wheelchair command selection}

Fig. 4 (a) depicts the wheelchair navigation panel. A total of nine commands is considered for wheelchair locomotion: six for direction, two for step size (travel distance), and one for switching the panel. The user can increase or decrease the step-length (i.e., distance to travel in each command) for each wheelchair motion using the "Step++" and "Step- -." commands respectively, as per the navigational need to follow the track. The maximum step length is $1.64 \mathrm{~m}$ and minimum is $0.36 \mathrm{~m}$. The switch command is used to switch between the exoskeleton control panel (ECP) and the wheelchair control panel (WCP). The searching of the commands on the monitor was done by POG and selection was made through the duration of eye fixations, which is called the dwell time. The $2 \mathrm{~s}$ dwell time was considered during the whole experiment. Once command button was pressed user can see the visual feedback

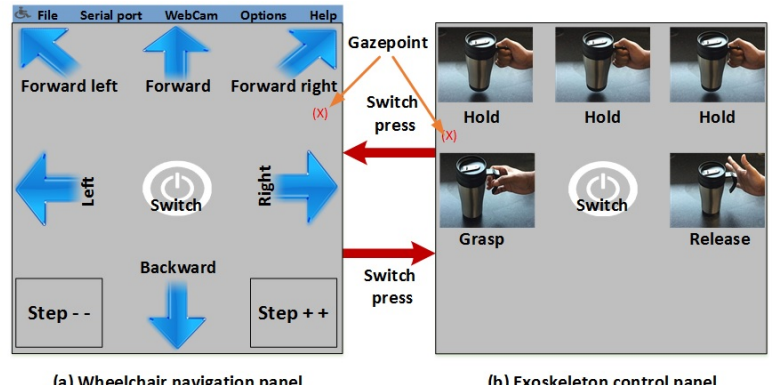

(a) Wheelchair navigation panel

(b) Exoskeleton control pane

Fig. 4. Combined Control Panels. (a) WCP, (b) ECP.

and control panel sends the command to the Arduino board using RS232 communication protocol, where an ATmega128 microcontroller based embedded system generates the control signals for wheelchair motion.

\section{B. Hand Exoskeleton Command Selection}

Fig. 4 (b) shows the hand-exoskeleton control panel. Three commands (a grasp, a hold, and a release) are considered for controlling the hand-exoskeleton and one for switching the panel. The operation of this switch command is the same as the WCP. The POG and dwell time were used for the searching and selection of the command. Once the user pressed the command button, the ECP sends the command to the embedded controller via the same way mentioned in section III (A), to initiate grasp, hold, and release actions in the exoskeleton. As the hold command needs a quick execution following a grasp, therefore we have also given the facility to execute this command as soon as the user withdraws his/her gaze from any of the three command buttons, without the intervention of dwell time. This strategy has proven to be convenient for the users which we will discuss later in the result section.

\section{Algorithmic flow chart of the proposed system}

The algorithmic flow chart in Fig. 5 shows how the control panel takes a decision, i.e., which command needs to be executed, and when. There is a process in the algorithm which continuously updates the user inputs from the eye-tracker, and checks whether the dwell time is over. The WCP and ECP are flipped from one to the other by taking note of the current panel (CP) and whether the switch button (Button-Coordinate $(B C)=6)$ is pressed. There is a process inside the algorithm which looks for the button co-ordinates and determines the specific command associated with the button. A button at the same coordinate, can act for different commands in WCP and ECP. For example the button at the top middle acts as forward command in WCP and hold in ECP. Once a command is executed, the corresponding button for that command turns green to display a visual feedback to the user.

\section{Performance metrics for evaluation}

Several performance metrics were set to establish the feasibility of the system and its stability in the test environments 


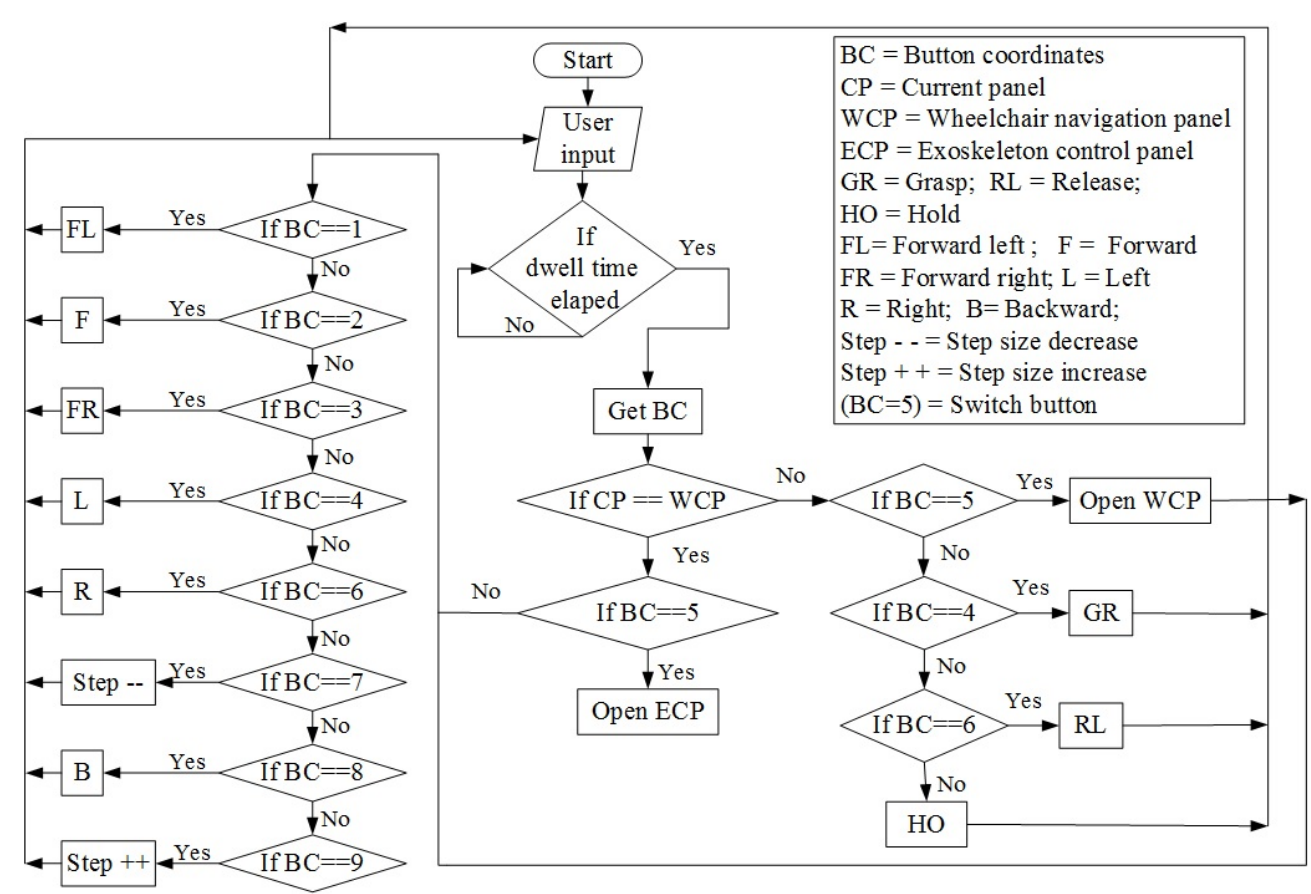

Fig. 5. Algorithmic flowchart of the proposed system.

(i.e., track complexity, cf. Fig.2). We have considered the following metrics to evaluate the test outcomes:

1) Total number of interactions: The total number of different commands executed during one experiment is denoted by TNI. Usually, a difficult track requires more manipulations leading to more interactions in terms of command execution.

$$
T N I=\sum_{i=1}^{N} \text { Count }_{\text {comm }_{i}}
$$

where $N$ is the total number of possible commands in both panels, 8 in WCP, 3 in ECP, 1 common between WCP and $\operatorname{ECP}(N=12)$, comm $_{i}$ is a particular command and Count $_{\text {comm }_{i}}$ is the total count of the comm $_{i}$ during one particular experiment.

2) Lap completion time: The average lap completion time ( $\mathrm{T}$ in min) was measured with respect to track complexity. The lap length ( $\mathrm{L}$ in $\mathrm{m}$ ) of the simple and complex track was $15.3 \mathrm{~m}$ and $16.02 \mathrm{~m}$, respectively (see in Fig. 2). Although there is not much difference in lap length, the number of turns in the complex track is more than the simple one which increases the difficulty level of the test.

$$
T=\frac{1}{60} \sum_{j=1}^{T N I} t_{j}
$$

where $t_{j}$ is the time interval between two successive interactions in terms of command selection.

3) Wheelchair speed: The speed ( $\mathrm{S}$ in $\mathrm{m} / \mathrm{min}$ ) is calculated based on the total path length the user has traversed while following the track and is defined as:

$$
S=\frac{1}{T} \sum_{i=1}^{D C} \sum_{j=1}^{T N I_{i}} S t p_{i j}
$$

where, $D C$ is the total number of directional commands $(D C=6)(\mathrm{F}, \mathrm{B}, \mathrm{L}, \mathrm{R}, \mathrm{FL}$, and $\mathrm{FR})$ and $T N I_{i}$ is the number of $\mathrm{t}$ of these commands during a test. $S t p_{i j}$ is the step-length associated with each of these commands.

4) Information Transfer rate: The information transfer rate (ITR in bit/min) of the eye-tracking control [24], [25], [26], [27] was measured to validate the system performance across the number of possible different outputs, with $V=\log _{2}(N)$.

$$
I T R=\frac{60 \cdot T N I \cdot V}{\sum_{j=1}^{\mathrm{TNI}} t_{j}}
$$

\section{RESUlts}

The results are obtained from the user interaction log recorded during each test. A total of 16 tests were conducted, wherein 8 tests are done with 8 naive participants on the simple track and the other 8 tests were conducted with the rest of the 8 naive participants on the complex track. Therefore, we had 8 user interaction logs from each of the tracks after the end of the experiment. In every log-file we recorded the commands executed by the participant throughout the test duration, along with the execution time and the step-size associated with each navigation command. The data, stored in the log-files, are then used to calculate different performance metrics for the evaluation of each test. To analyze the feasibility of the system we have used the one sample t-test on the performance metrics 


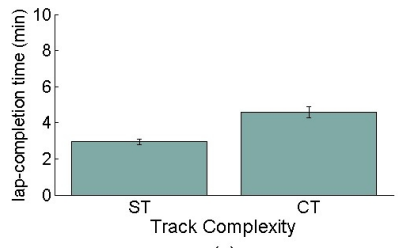

(a)

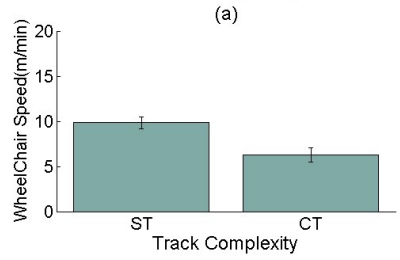

(c)

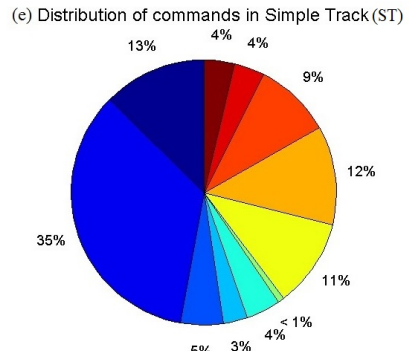

$5 \% 3 \%$

Fig. 6. Different performance indices in simple Track (ST) and complex track (CT). (a) Lap-completion time, (b) Total number of commands, (c) Wheelchair speed, (d) ITR. (e)-(f) Distribution of total number of commands for simple track (e) and complex track (f).

across the participants of a group to accept the null-hypothesis for mean, which states that sample mean and the population mean are similar. This means that for a given particular task complexity, if the performance among the naïve users turns out to be similar, then the system is feasible for use. To find out the stability of the system performance, we applied twosample t-test to see the change in the performance metrics across the task complexities. The idea behind this is to validate that the metrics used to calculate the system performance are legitimate. The mean and standard error of mean (SEM) for each performance metrics: lap-completion-time, number of commands, wheelchair speed, and the ITR, are shown in Fig. 6 (a)-(d) respectively, for each task complexity. Fig. 6 (e)-(f) show the distribution of the commands as a percentage of the total number of commands used by the participants for the simple and complex track.

The mean lap-completion time in simple and complex track is found to be $2.94 \pm 0.01 \mathrm{~min}$ and $4.57 \pm 0.31 \mathrm{~min}$ respectively, while the total number of commands used in each of these test conditions are $40.63 \pm 2.05$ and $57.75 \pm 3.40$ respectively. The mean wheelchair speed for the simple and complex track conditions is $9.85 \pm 0.65 \mathrm{~m} / \mathrm{min}$ and $6.26 \pm 0.82 \mathrm{~m} / \mathrm{min}$. The ITR is found to be $55.28 \pm 1.29 \mathrm{bits} / \mathrm{min}$ and $51.02 \pm 1.72$ bits/min for the simple and complex test conditions respectively. In the command distribution pie chart, we observe that the forward command was most frequently used (average $35 \%$ in simple track and average $34 \%$ in complex track) by the user as compared to the other commands. The left and right buttons were used on an average 3\% and $4 \%$ in simple track respectively, while these buttons were used $6 \%$ and $6 \%$ respectively in the complex track. The stepup command was used $12 \%$ in simple track and $10 \%$ in complex track while the step-down command was used $9 \%$ and $12 \%$ in simple and complex track respectively. The one sample t-test on the performance metrics applied for each track complexity yielded $\mathrm{p}<0.01$, which highlights the feasibility of the system. Moreover, a two-sample t-test was applied to compare the performance metrics between the simple and complex test conditions. The lap-completion time and the total number of commands are significantly lower in simple track than the complex track $(\mathrm{p}<0.01)$, while the wheelchair speed is significantly higher $(\mathrm{p}<0.01)$. Finally, the ITR is not significantly different between the two test conditions. This shows that the lap-completion time, the number of commands, and the wheelchair speed can serve as reliable metrics to relate the system performance in relation to the task complexity.

\section{DISCUSSION}

EMOHEX is the first example of a wheelchair mounted hand-exoskeleton device fully operated by POG using a lowcost eye-tracking device. Unlike the wheelchair mounted robotic arms, this device has the potential to increase the user's motivation as it will feel like they are using their own arm for doing ADLs. The POG being the input modality is useful, as the oculomotor system often remains functioning normally for people suffering from neuro-muscular disabilities. Moreover, the EMOHEX is a low cost device. It can be easily integrated with any wheelchair. In fact, the EMOHEX system was validated on the basis of testing its feasibility and stability, by analyzing its performance on the basis of task complexity using appropriate performance metrics. The results clearly show the consistency of the different functionalities of EMOHEX. The performance across participants was stable for both tasks. The lap-completion time and the number of commands used were significantly higher in the complex track than in the simple track, while the wheelchair speed was found to be lower for the complex track. This is intuitive in the sense that for the complex track the user needs to face more twisted turns, which require a higher number of commands for navigation. This would lead to lower the wheelchair speed and increase the lap-completion time. Yet, the ITR was found to be unaffected by the task complexity, which shows the robustness of the system. In addition, we found that left and right commands were used almost doubly frequent by the user while traversing the complex track, which is reasonable as the complex track includes more turns. All the users have successfully grabbed, held, and dropped the object in the bin using the ECP, and the grab-and-release commands were used in almost equal proportion. However, the hold buttons were not pressed by any of the users as it was convenient for them to not focus on any buttons in the ECP to initiate the hold action quickly. These results also show that integration of the exoskeleton and the wheelchair with the eye-tracker is successful as users didn't 
find any complicacy in using the WCP and ECP alternatively whenever necessary. This switching method is also very crucial for enhanced ITR, as the command button locations in the control panel GUI are unaltered. Furthermore, it facilitates the augmentation of additional control panels while keeping the same GUI format, if additional robotic devices are to be integrated with the existing system. Future works will include the addition of proximity sensors to avoid colliding with obstacles, and reject any unsafe command issued by the user. Finally, while the current functionalities of EMOHEX focus on ADL and not directly on rehabilitation, modifications of the system such as operating the exoskeleton in assist-as-needed mode or replacing the dwell time based command selection with a BCI can provide a multimodal device for rehabilitation purposes.

\section{CONCLUSION}

This paper describes how an eye-tracker based robotic platform can be implemented for controlling a powered wheelchair mounted hand-exoskeleton to cater to the ADL of people suffering from a severe disability of lower and upper-extremities. It uses two interchangeable control panels with unaltered command button distribution for the ease of operability. The experimental results have shown the performance of the eyetracker based HCI to be reliable and user friendly. The tests, conducted on the different track complexities have shown the feasibility and stability of the system quite comprehensively, as all the participants satisfactorily completed the task. Overall EMOHEX is a promising device to improve the quality of living of the disabled people and also have the prospect of encompassing larger arena of assistive technologies in the future.

\section{ACKNOWLEDGMENT}

Y.K.M. is supported by Govt. of India (Education11016152013). G.P., K.W.-L., and H.C. are supported by the Northern Ireland Functional Brain Mapping Facility (1303/101154803). GP, A.C., S.S.N, and A.D. are supported by DST-UKIERI thematic partnership project A BCI Operated Hand Exoskeleton based Neurorehabilitation System for Movement Restoration in Paralysis (DST-20130354).

\section{REFERENCES}

[1] A. J. Skalsky and C. M. McDonald, "Prevention and management of limb contractures in neuromuscular diseases," Phys Med Rehabil Clin $N A m$, vol. 23, no. 3, pp. 675-687, 2012.

[2] R. E. Cowan, B. J. Fregly, M. L. Boninger, M. Chan, M. M. Rodgers, and D. J. Reinkensmeyer, "Recent trends in assistive technology for mobility," J. of NeuroEng. Rehab.(JNER), vol. 9, no. 20, pp. 1-8, 2012.

[3] C. S. Lin, C. W. Ho, W. C. Chen, C. C. Chiu, and M. S. Yeh, "Powered wheelchair controlled by eye-tracking system," Optica Applicata, vol. 36, no. 2-3, pp. 401-412, 2006.

[4] R. Maskeliunas and R. Simutis, "Multimodal wheelchair control for the paralyzed people," Elektronika ir Elektrotechnika, vol. 5, no. 111, pp. 81-84, 2011.

[5] J. S. Nguyen, S. W. Su, and H. N. Nguyen, "Experimental study on a smart wheelchair system using a combination of stereoscopic and spherical vision," in Proc. of the 35th Int. Conf. of the IEEE Engineering in Medicine and Biology Society (EMBC), 2013, pp. 4597-4600.
[6] S. I. Ktena, W. Abbott, and A. A. Faisal, "A virtual reality platform for safe evaluation and training of natural gaze-based wheelchair driving," in Proc. of the 7th Annual International IEEE EMBS Conference on Neural Engineering Montpellier, 2015, pp. 236-237.

[7] P. Biswas and P. Langdon, "A new input system for disabled users involving eye gaze tracker and scanning interface," Journal of Assistive Technologies, vol. 5, no. 2, pp. 58-66, 2011.

[8] Y. K. Meena, G. Prasad, H. Cecotti, and K. Wong-Lin, "Powered wheelchair control with a multimodal interface using an eye-tracking and a soft-switch," in Proc. of 7th Translational Medicine Conf., 2015.

[9] A. Frisoli, C. Loconsole, D. Leonardis, F. Banno, M. Barsotti, C. Chisari, and M. Bergamasco, "A new gaze-bci-driven control of an upper limb exoskeleton for rehabilitation in real-world tasks," Systems, Man, and Cybernetics, IEEE Transactions on, vol. 42, no. 6, pp. 1169-1179, 2012.

[10] S. Deng, J. A. Kirkby, J. Chang, and J. J. Zhang, "Multimodality with eye tracking and haptics: a new horizon for serious games?" International Journal of Serious Games, vol. 1, no. 4, pp. 17-33, 2014.

[11] S. K. Banala, S. K. Agrawal, S. H. Kim, and J. P. Scholz, "Novel gait adaptation and neuromotor training results using an active leg exoskeleton," Mechatronics, IEEE/ASME Transactions on, vol. 15, no. 2, pp. 216-225, 2010.

[12] A. U. Pehlivan, F. Sergi, and M. K. OMalley, "A subject-adaptive controller for wrist robotic rehabilitation," Mechatronics, IEEE/ASME Transactions on, vol. 20, no. 3, pp. 1338-1350, 2015.

[13] J. Ueda, D. Ming, V. Krishnamoorthy, M. Shinohara, and T. Ogasawara, "Individual muscle control using an exoskeleton robot for muscle function testing," Neural Systems and Rehabilitation Engineering, IEEE Transactions on, vol. 18, no. 4, pp. 339-350, 2010.

[14] C. L, F. Wang, R. Morrison, N. Sarkar, and D. G. Kamper, "Design and development of the cable actuated finger exoskeleton for hand rehabilitation following stroke," Mechatronics, IEEE/ASME Transactions on, vol. 19, no. 1, pp. 131-140, 2014.

[15] P. Maciejasz, J. Eschweiler, K. Gerlach-Hahn, A. Jansen-Troy, and S. Leonhardt, "A survey on robotic devices for upper limb rehabilitation," J. of NeuroEng. Rehab.(JNER), vol. 11, no. 3, pp. 1-29, 2014.

[16] J. d. R. Millán, R. Rupp, G. R. Müller-Putz, R. Murray-Smith, C. Giugliemma, M. Tangermann, C. Vidaurre, F. Cincotti, A. Kbler, R. Leeb, C. Neuper, K. R. Muller, and D. Mattia, "Combining braincomputer interfaces and assistive technologies: state-of-the-art and challenges," Frontiers in neuroscience, vol. 4, no. 161, pp. 1-15, 2010.

[17] A. Chowdhury, H. Raza, A. Dutta, and G. Prasad, "Cortico-muscularcoupling and covariate shift adaptation based bci for personalized neurorehabilitation of stroke patients," in Proc. of BCI Meeting, 2016, p. 136.

[18] R. Rupp, "Challenges in clinical applications of brain computer interfaces in individuals with spinal cord injury," Frontiers in neuroengineering, vol. 7, no. 38, pp. 1-15, 2014.

[19] D. Rathee, H. Cecotti, and G. Prasad, "Estimation of effective frontoparietal connectivity during motor imagery using partial granger caisality analysis," in Proc. of the Int. Joint Conf. on Neural Networks, 2016.

[20] P. Majaranta and A. Bulling, Eye Tracking and Eye-Based HumanComputer Interaction, ser. Advances in Physiological Computing. Springer, 2014, pp. 39-65.

[21] F. Marcela, L. Figueiredo, P. Novk, O. Stepankova, and A. Gomes, "Hands-free interaction with a computer and other technologies," Universal Access in the Info. Society, vol. 8, no. 4, pp. 277-295, 2009.

[22] "The eye tribe, copenhagen, denmark," https://theeyetribe.com/, accessed: 2015-06-01.

[23] E. S. Dalmaijer and T. Building, "Is the low-cost eyetribe eye tracker any good for research," PeerJ Prepr., pp. 1-35, 2014.

[24] D. J. McFarland, W. Sarnacki, and J. R. Wolpaw, "Brain-computer interface (BCI) operation optimizing information transfer rates," Biol. Psychol., vol. 63, no. 3, pp. 237-251, Jul. 2003.

[25] D. O'Doherty, Y. K. Meena, R. Haider, H. Cecotti, and G. Prasad, "Exploring gaze-motor imagery hybrid brain-computer interface design," in Proc. of the IEEE Int. Conf. on BIBM, 2014, pp. 335-339.

[26] Y. K. Meena, H. Cecotti, K. Wong-Lin, and G. Prasad, "Towards increasing the number of commands in a hybrid brain-computer interface with combination of gaze and motor imagery," in Proc. of the 37th Annual Int. Conf. of the IEEE EMBC, 2015, pp. 506-509.

[27] Y. K. Meena, H. Cecotti, K. Wong-lin, and G. Prasad, "Simultaneous gaze and motor imagery hybrid bci increases single-trial detection performance: a compatible incompatible study," in Proc. of the IEEEEMBS 9th Int. Summer School in Biomedical Signal Processing, 2015, p. 1 . 